DOI https://doi.org/10.18551/rjoas.2016-11.21

\title{
STUDY OF VEGETABLE CROP PEELING BY BRUSH WORKING HEAD
}

\author{
Aliyev S.H., Researcher \\ Azerbaijan Technological University, Azerbaijan \\ E-mail: shakiraliyev@mail.ru
}

\begin{abstract}
It is set a challenge of constructing a dynamic model of the brush working head of the washing and cleaning machine in the preparation of vegetable tubers on the line of canned vegetables production. They are specified conditions of forming of an array of lint brush under the action of forces applied to it during operation. It is compiled the design scheme of interaction between the working head and the process material. Based on the prepared scheme they are determined formulas determining the forces acting on the brush working head. The physical meaning of these relations is determined. It has been established that there is an additional frictional force influencing on the working head. This force is caused by the elastic deformation and discontinuities in the organ of the working head. This force depends on the geometrical parameters, namely on the angle of inclination of the process material against the horizontal and the inclination angle of friction force between the rib and the top-side of the lint characterizing deformations and discontinuities. The resulting formulas are basic relations describing the ratio of brush working heads with cleaned vegetable tubers.
\end{abstract}

\section{KEY WORDS}

Vegetable, root, purification, peeling, dynamic model, working head, friction.

One of the main technological processes of canned vegetables production is the raw materials washing and purification, as well as its peeling. Vegetables at the canning enterprises can be contaminated with soil, sand and dust. All raw materials are washed thoroughly in cold running water until whole removal of mechanical impurities and partial microorganisms' removal.

For purification of vegetable raw materials (carrot and red beet) they are treated in hot caustic solutions followed by washing in a drum washing machine with water supplied under pressure.

When leaving the pressurized zone a peel is broken because of moisture selfevaporation in the subcutaneous layer, and then it is separated in the washing and cleaning machine under the action of rotating brushes and water jets [1, 2].

In these machines, the brush working heads, being final assembly, play an important role in ensuring the requirements imposed on the process. However, insufficient knowledge of the process is a limiting factor in its structural and technological improvement. Thus consideration of the basis of calculation of these working heads on the line of root vegetables peeling is an urgent task.

\section{RESEARCH METHODOLOGY}

Workflow of any brush is based on the friction between its working surface and cleaning organ. However, neither the classical theory of dry friction or additions made to it, do not apply to the investigation of the dynamic interaction between the brush and the purifying object. Great efforts on friction [3-5] are based on the assumption that the surface of each of the friction organs always has nature of a continuous surface. Meanwhile, during the interaction with the purified material working brush surface may experience large elastic deformation. Therefore, they cannot be ignored additional forces of friction between the brush surface and roots treated by it, since they are not small compared with the adhesive and hysteresis tribonic forces, the combined effect of which can be estimated using the dry 
friction coefficient. It must be taken into account that the elastic deformation of the reaction of deformed brush pile stipulated only by normal pressure.

Purifying root can be intruded in villi array, not only deforming the working surface of the brush, but also forming breaks in it. Therefore it ceases to be a continuous surface and can not be replaced when the construction of a dynamic model of interaction between the root brush and geometric continuous surface. These well-known experimental data relating to the brush working heads, contrary to the proposals, underlying Coulomb tribonics and its subsequent modifications $[6,7]$.

It follows that for the study of the working process performed by brush working heads of washing and cleaning machine, classical theory of dry friction should be taken into account when one of the friction organs can be broken or deformed.

\section{RESULTS AND DISCUSSION}

It is necessary to develop a dynamic model of the brush working head (Figure 1).

Let $\mathrm{F}$ is a body of array of flat brush villi, which is limited by two parallel planes $\psi 0$ and $\psi$, the distance between them is continuous in the absence of external load; the body consists of a cylindrical fibers (rods and villi) that satisfy the following conditions: a) under the action only of gravitational forces all fibers are right circular cylinders congruent to one another, their center lines are perpendicular to the planes $\psi 0$ and $\psi ; b$ ) one of the bases of each villi (lower) is cantilevered in the plane $\psi 0$, the other (the free end) lies in the plane $\psi$; c) the villi distribution density is constant (i.e. if $\Omega$ is a bounded simply connected domain in the plane $\psi 0, v(\Omega)$ - the number of villi, the lower ends of which are fixed inside the domain $\Omega$, and $\Omega 1$ and $\Omega 2$ - arbitrary equal figures in the plane $\psi 0$, then $v(\Omega 1)=v(\Omega 2)$; d) all of the fibers are made of the same material and have the same density, that is why elastic constant (Young's modulus and Poisson's ratio) are the same for all of the villi; e) villi are not rigidly connected together and their mechanical impact on one another can be carried out only by contact; f) fibers test elastic deformation; g) under the action of external forces and corresponding support reactions each villus behaves like a thin rod.

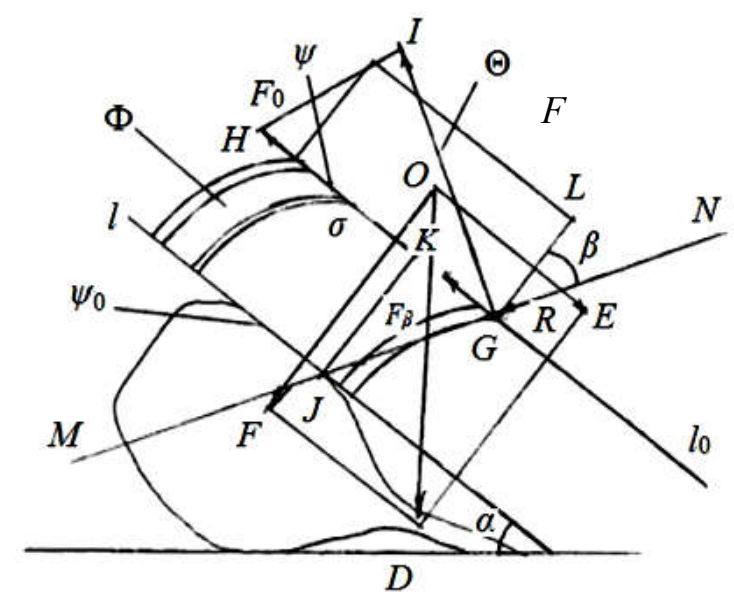

Figure 1 - The forces acting on the brush working head

Define $\mathrm{F}$ body position in space. To do this, it is necessary to select arbitrary fixed horizontal plane $\mathrm{H}$ as a basic system. They are given: line I, where planes $\psi 0$ and $\mathrm{H}$ are intercrossed; an acute angle between them $(\alpha ; 0<\alpha<\pi / 2)$, plane $\psi 0$ lies below the plane $\psi$. Thereby position of the body $\mathrm{F}$ is clearly defined.

Examine the body $\Theta$ :

1. It has the shape of a rectangular parallelepiped disposed so that parallelepiped $\Theta$ rests its face $(\sigma)$ on the plane $\psi$, which is a unilateral geometric relationship, superimposed on the body $\Theta$ ); parallelepiped $\Theta$ is located in the initial position above the line $\mathrm{I}$, and any of its edges is parallel or perpendicular to it. 
2. Roughness of plane sides facing one to the other and of the edge $\psi$ is uniform, that is, none of the possible direction of the body $\Theta$ slip on the plane $\psi$ does not stand out among the other.

3. The body $\Theta$ is homogeneous.

4. Between bodies $\mathrm{F}$ and $\Theta$ only dry friction forces act (indicate coefficient of friction and the angle $f$ and $\varphi$ respectively).

5. The main point of the system $S$ of the active forces applied to the body $\Theta$ is equal to the zero vector, and its main vector $\vec{F}$ has the symmetry center $O$ of the parallelepiped $\Theta$ as the point of application. It acts in the plane $\Theta$ perpendicular to the line I and passes through the point $\mathrm{O}$.

6. If the resultant $\vec{F}$ is decomposed into components $\vec{v}$ and $\vec{\tau}, \vec{v}$ is normal and is $\vec{\tau}$ coplanar to the plane $\psi$, then the vector forms an acute angle with the gravity direction, i.e. the force $\vec{F}$ pushes the body $\Theta$ to the plane $\psi$.

7. The length of the side edge of the parallelepiped $\Theta$ is sufficiently small, so that the tilting moment caused by the force $\vec{\tau}$ can be neglected without any results accuracy sacrificing.

From conditions $1 . . .7$ implies that the rotational movement of the body $\Theta$ is impossible. It may rest with respect to the plane $\varphi 0$, slide along it, or introduced into the body $F$ (driving while progressively). Suppose that the following conditions are met: penetration of the parallelepiped $\Theta$ in the body $\mathrm{F}$ can be regarded in the quasi-static approximation (i.e. they can be neglected inertia forces generated by the body $\Theta$ movement, lies in its implementation in the body $\mathrm{F}$, while accuracy of the results will be sufficient); at penetration of the parallelepiped $\Theta$ in the body $F$ its face ( $\sigma)$ is parallel to its initial position, and the section of the parallelepiped $\Theta$ of arbitrary plane, perpendicular to the line I, moves, staying in the plane $\Theta$.

With the help of a mechanical system consisting of bodies $F$ and $\Theta$, it can be described with a high degree of accuracy the brush interaction with root crop (its role plays the body $\Theta$ during its workflow).

Breaks in the body $\mathrm{F}$, caused by the parallelepiped $\Theta$ penetration are stipulated by the buckling of the villi, which receive impact of the body $\Theta$ by their free ends. We call these villi as carriers. When moving the body $\Theta$ (paragraph 1), some carrier villi returns to the original (undeformed) position and other villi raised by the parallelepiped edge ( $\sigma$ ) become carriers.

Let $I_{0}$ be the line closest to the line I. Buckling of bearing villi leads to the fact that loose ends experience movement, directed to the plane $\psi 0$. It entails the formation of contact between the rib $I_{0}$ and the side surfaces of the villi, located directly below carriers (in the figure it is given section of bodies $\mathrm{F}$ and $\Theta$ of the plane $\Theta$ : rib $I_{0}$ is projected to a point $\mathrm{G}$, and the villi contacting with it, to a villus with loose end $R$ ). It raises the impact force of the purifying object on villi, side surfaces of which contact with side $\mathrm{I}_{0}$.

We determine the system of forces applied to villi. Let $m$ be its mass. Suppose that the only active external force acting in the considered mechanical system is the weight $\mathrm{mg}$. We expand it to normal to the plane $\psi$ and to components $\vec{v}, \vec{\tau}$, coplanar to it. According to experimental data, the power $\vec{v}$ is balanced by a set of elastic reaction generated by buckling of bearing villi. Therefore only the force $\vec{\tau}$ is applied to the villi, side surfaces of which are in contact with the edge $\mathrm{I}_{0}$. If $\mathrm{P}$ is a number of these villi, then a force $\frac{\vec{\tau}}{P}$ coplanar to geometrical axis [8] corresponding to the villi acts on any of them. This force causes the flat bending of the villi, which geometric axis becomes curved and elastic villi line [9]. Coulomb friction forces acts between the side surface of each of these villi and rib $I_{0}$. These forces under the conditions ( $\mathrm{g}$ ) can be considered as applied along the tangent to the elastic villi line in the direction opposite to the movement of the body $\Theta$.

Select any of the villi, the side surfaces of which come into contact with an edge $I_{0}$. Condition (g) suggests that contact occurs at a single point; denoted it by G. We can also 
assume, while maintaining high accuracy that villus coincides with the elastic line. The angle between the tangent to the elastic villi line drawn at the point $G$ and passing through the edge $I_{0}$ perpendicular to the side edge $(\sigma)$ of the parallelepiped $\Theta$ is the same for each of the villi that are in contact with the side $I_{0}$ (in the figure - angle NGL); this angle is denoted by $\beta$; $0 \leq \beta \leq \frac{\pi}{2}$. $\beta=$ const at the equilibrium position of the considered mechanical system.

Movement of the body $\Theta$ is limited only by frictional forces between the face $(\sigma)$ and the upper bearing of slice villi, as well as between the rib $I_{0}$ and the side surfaces of the villi touching it. Let the principal vectors of these two forces be $\vec{F}_{0}$ and $\vec{F}_{\beta}$ respectively (in the figure $\vec{F}_{\beta}=\vec{G} J$ ). It follows from conditions (a, b, c, g) and (5) that the forces $\vec{F}_{0}$ and $\vec{F}_{\beta}$ act in the plane $\Theta$, because it is located center of each of these parallel power systems in it.

Let $\left(\vec{F}_{\beta}\right)_{\sigma}$ is the projection of the vector $\vec{F}_{\beta}$ on the plane $\sigma$. A necessary and sufficient condition for static equilibrium of the body $\Theta$ is an inequality:

$$
\tau \leq F_{0}+\left(\vec{F}_{\beta}\right)_{\sigma} .
$$

According to the theorem on the angles with mutually perpendicular sides $D \mathscr{G}=\alpha$. Consequently, $D \Theta E=\frac{\pi}{2}-\alpha$.

In the triangle $D O E|O D|=m g,|O E|=\tau$, therefore

$$
\tau=m g \sin \alpha
$$

According to Coulomb's law the friction force

$$
F_{0}=f v .
$$

In the triangle DOE $|O F|=v$, therefore

$$
v=m g \cos \alpha .
$$

Formulas (3) and (4) imply

$$
F_{0}=m g f \cos \alpha
$$

As $L \xi_{N}=\beta$, then $K \xi_{J}=\frac{\pi}{2}-\beta$, consequently the triangle GJK imply

$$
\left(\vec{F}_{\beta}\right)_{\sigma}=F_{\beta} \sin \beta \text {. }
$$

From the part of the body $\Theta$ a system of parallel forces with resultant $\vec{\tau}$ is applied to the villi that are in contact with the edge $\mathrm{I}_{0}$. Its point of application is the intersection of the plane $\Theta$ with the edge $I_{0}$ (in the figure - point $G$ ). We expand the force $\vec{G} H=\vec{\tau}$ in two components acting in the plane $\Theta$, one of which is perpendicular to the line $\mathrm{MN}$ (in the figure $\vec{G} I$ ), the other is collinear [10] to it and is directed towards the displacement of the body $\Theta$, that is from $\mathrm{G}$ to $\mathrm{N}$ (this power is not given in the figure because we do not use it in the future). From triangle IGH (in which $|G H|=\tau$ ) it follows that $|G I|=\tau \cos \beta$. Since the $\vec{G} I$ force of normal pressure of the body at $\Theta$ villi that are in contact with the edge $\mathrm{I}_{0}$ we have 


$$
F_{\beta}=f \tau \cos \beta .
$$

Formulas (2) and (7) imply

$$
F_{\beta}=m g f \sin \alpha \cos \alpha \text {. }
$$

Formulas (6) and (8) imply

$$
\left(F_{\beta}\right)_{\sigma}=\frac{1}{2} m g f \sin \alpha \sin 2 \beta .
$$

According to relations (2), (5) and (9), the condition (1) becomes

$$
m g \sin \alpha \leq m g f \cos \alpha+\frac{1}{2} m g f \sin \alpha \sin 2 \beta .
$$

From this it follows that

$$
\sin \alpha \leq f \cos \alpha+\frac{1}{2} f \sin \alpha \sin 2 \beta
$$

Since $0<\alpha<\frac{\pi}{2}$, we have $\cos \alpha>0$. From this it follows that division of both sides of inequality (11) by $\cos \alpha$ leads to the equivalent inequality. After division it will be

$$
\begin{array}{r}
\operatorname{tg} \alpha \leq f\left(1+\frac{1}{2} f \operatorname{tg} \alpha \sin 2 \beta\right), \\
\text { or } \\
\operatorname{tg} \alpha \leq \operatorname{tg} \varphi(1+\operatorname{tg} \alpha \sin 2 \beta) .
\end{array}
$$

Establish the physical meaning of these relations. To this end, we set $\beta=0$ in the inequalities (12) and (13) (i.e. suppose that the body $F$ does not experience any deformations or ruptures, resulting tribonic interaction of bodies $\Theta$ and $F$ takes place in accordance with the Amontons-Coulomb laws [11]. Formulas (12) and (13) be $\operatorname{tg} \alpha<1, \operatorname{tg} \alpha \leq \operatorname{tg} \varphi$.

It follows that $\frac{1}{2} m g f \sin \alpha \sin 2 \beta$ is the module of additional friction force acting on the body $\Theta$ and caused by elastic deformations and breaks in the body $F$. This force depends on the geometric parameters $\alpha$ and $\beta$, characterizing these deformations and breaks. Represent relations (12) and (13) in the following form:

$$
\begin{aligned}
& \left(1-\frac{1}{2} f \sin 2 \beta\right) \operatorname{tg} \alpha \leq f \\
& \left(1-\frac{1}{2} f \sin 2 \beta\right) \operatorname{tg} \alpha \leq \operatorname{tg} \varphi .
\end{aligned}
$$


As $0 \leq \frac{1}{2} f \sin 2 \beta=\frac{1}{2} \operatorname{tg} \varphi \sin 2 \beta<1$ we have

$$
1-\frac{1}{2} f \sin 2 \beta>0
$$

or equivalently

$$
1-\frac{1}{2} \operatorname{tg} \varphi \sin 2 \beta>0
$$

From inequalities (16) and (17) it follows that by division of both sides of (14) and (15) by $1-\frac{1}{2} f \sin 2 \beta$ and $1-\frac{1}{2} \operatorname{tg} \varphi \sin 2 \beta$ the inequality signs remain unchanged, thus

$$
\begin{aligned}
& \operatorname{tg} \alpha \leq \frac{f}{1-\frac{1}{2} f \sin 2 \beta} \\
& \operatorname{tg} \alpha \leq \frac{\operatorname{tg} \varphi}{1-\frac{1}{2} \operatorname{tg} \varphi \sin 2 \beta}
\end{aligned}
$$

Conclusion. Formulas (18) and (19) are the main relationships describing the interaction of brush working heads with purifying tubers intended for processing into canned food products.

\section{REFERENCES}

1. Dragileva A.I., Drozdov V.S. Technological equipment of processing enterprises in AIC. Moscow, 2001, 352 p.

2. Nazarov A.I., Fan-Jung A.F. The technology of fruit and vegetable preserves. Moscow, 1981. 240 p.

3. Rozenblat G.M. Dynamical systems with dry friction. Izhevsk. 2066. 203 p.

4. Frolov K.V. Modern tribology: Results and Prospects. Moscow, 2014. 480 p.

5. Musalimov V.M. et al. Basics of tribology. Saint-Petersburg, 2009. 77 p.

6. Alekseyev G.V., Logunov V.S. Features of contact interaction of the friction surfaces. In Tekhnika mashinostroyeniya, 2001, No. 1, p. 84-92.

7. Dzhonson K. Contact mechanics. Moscow, 1980, 509 p.

8. Vygodskiy M.Y. Handbook of higher mathematics. Moscow, 2010. 384 p.

9. Dolgov N.I. On calculation of the bending of the small stiffness rods. In Strength Calculations. Moscow, 1963. P. 25-42.

10. Bugrov Y.S., Nikolsky S.M. Higher Mathematics, Volume One: Elements of linear algebra and analytic geometry. Moscow, 2004, 288 p.

11. Mur D. Fundamentals and application of tribonics; translate from English. Moscow, 1978, $483 \mathrm{p}$. 